Kragujevac Journal of Mathematics

Volume 40(2) (2016), Pages 272-279.

\title{
REMARKS ON THEOREMS FOR CYCLIC QUASI-CONTRACTIONS IN UNIFORMLY CONVEX BANACH SPACES
}

\author{
N. V. DUNG ${ }^{1,2}$ AND S. RADENOVIĆ ${ }^{3,4, *}$
}

\begin{abstract}
In this note we show an error in the proof of [1, Theorem 2.3]. Then we give a counterexample to show that theorems for cyclic quasi-contractions in [1] are not true. Also the proofs of theorems for cyclic strongly quasi-contractions in that paper are not true. We also state the revisions with modified conditions for main results in [1].
\end{abstract}

\section{INTRODUCTION}

The Banach contraction principle is a fundamental result in fixed point theory. Several extensions and applications in nonlinear analysis and optimization of this result were stated, see $[2-4,9,10,14]$ and the references given there. An interesting extension was proved by Kirk et al. [11] by using a cyclic condition, where the cyclic contraction is that deals with maps of the type $T: A_{i} \longrightarrow A_{i+1}, i=1, \ldots, p$ with $A_{p+1}=A_{1}$ and its contractive assumption is restricted to pairs $(x, y) \in A_{i} \times A_{i+1}$.

Cyclic conditions were then studied by many authors. In 2010 Petric [13] extended many fundamental metric fixed point theorems in the literature to maps with certain cyclic contractions. Păcurar and Rus [15] presented fixed point theorems for cyclic $\varphi$-contractions that were then noted by Radenović in [16]. In 2011 Karapinar and Sadarangani $[7,8]$ considered fixed point theorems for cyclic weak $\phi$-contractions. In 2012 Chen [5] proved fixed point theorems for cyclic Meir-Keeler type maps in complete metric spaces. In 2013 Amini-Harandi [1] introduced a new class of maps, called cyclic strongly quasi-contractions, which contains the cyclic contractions as a

Key words and phrases. cyclic quasi-contraction, fixed point, uniformly convex Banach space. 2010 Mathematics Subject Classification. Primary: 47H10, 54E35. Secondary: 54H25.

Received: April 21, 2016.

Accepted: June 19, 2016. 
subclass and proved some convergence and existence results of best-proximity point theorems for cyclic strongly quasi-contraction maps.

Definition 1.1 ([1], Definitions 2.2-2.3). Let $A$ and $B$ be nonempty subsets of a complete metric space $(X, d)$ and let $T: A \cup B \longrightarrow A \cup B$ such that $T(A) \subset B$ and $T(B) \subset A$. Then

(a) $T$ is called a cyclic quasi-contraction if for all $x \in A$ and $y \in B$ and some $c \in[0,1)$,

$$
\begin{aligned}
d(T x, T y) \leq & c \max \{d(x, y), d(x, T x), d(y, T y), d(x, T y), d(y, T x)\} \\
& +(1-c) d(A, B) ;
\end{aligned}
$$

(b) $T$ is called a cyclic strongly quasi-contraction if it is a cyclic quasi-contraction and for all $x \in A$ and $y \in B$

$$
d\left(T^{2} x, T^{2} y\right) \leq c d(x, y)+(1-c) d(A, B)
$$

The main results of [1] are as follows.

Theorem 1.1 ([1], Theorem 2.3). Let $A$ and $B$ be nonempty subsets of a metric space $X$ and let $T: A \cup B \longrightarrow A \cup B$ be a cyclic quasi-contraction map. For $x_{0} \in A \cup B$, define $x_{n+1}=T x_{n}$ for each $n \geq 0$. Then $\lim _{n \rightarrow \infty} d\left(x_{n}, x_{n+1}\right)=d(A, B)$.

Theorem 1.2 ([1], Theorem 2.4). Let $A$ and $B$ be nonempty subsets of a uniformly convex Banach space $X$ and let $T: A \cup B \longrightarrow A \cup B$ be a cyclic quasi-contraction map such that $A$ is convex. For $x_{0} \in A \cup B$, define $x_{n+1}=T x_{n}$ for each $n \geq 0$. Then $\lim _{n \rightarrow \infty}\left\|x_{2 n+2}-x_{2 n}\right\|=0$ and $\lim _{n \rightarrow \infty}\left\|x_{2 n+3}-x_{2 n+1}\right\|=0$.

Theorem 1.3 ([1], Theorem 2.5). Let $A$ and $B$ be nonempty subsets of a uniformly convex Banach space $X$ and let $T: A \cup B \longrightarrow A \cup B$ be a cyclic strongly quasicontraction map such that $A$ is convex. For $x_{0} \in A \cup B$, define $x_{n+1}=T x_{n}$ for each $n \geq 0$. Then for each $\varepsilon>0$ there exists $n_{0}$ such that for all $m>n \geq n_{0}$ we have $\left\|x_{2 m}-x_{2 n+1}\right\|<d(A, B)+\varepsilon$.

Theorem 1.4 ([1], Theorem 2.6). Let $A$ and $B$ be nonempty subsets of a uniformly convex Banach space $X$ and let $T: A \cup B \longrightarrow A \cup B$ be a cyclic strongly quasicontraction map such that $A$ and $B$ are convex. For $x_{0} \in A \cup B$, define $x_{n+1}=T x_{n}$ for each $n \geq 0$. Then there exists unique $x \in A$ such that $\lim _{n \rightarrow \infty} x_{2 n}=x, T^{2} x=x$ and $\|x-T x\|=d(A, B)$.

The author of [1] also posed the following question.

Question 1.1 ([1], Question 2.8). Does the conclusion of Theorem 1.4 remains true for cyclic quasi-contraction maps?

Unexpectedly, in the proof of Theorem 1.1, the author used the cyclic quasicontraction condition for the pair $\left(x_{i}, x_{j}\right)$ which may not belong to $A_{k} \times A_{k+1}$ in general, see the proof of (2.4) on [1, page 1669]. This is inappropriate since the cyclic 
quasi-contraction condition only holds for pairs in $A_{k} \times A_{k+1}$ for $k=1, \ldots, n$. The similar inappropriateness also appeared in the proof of [12, Theorem 4.1], see the inequality (4.2) on page 79 of that paper and see also [6]. Theorem 1.1 was then used in the proof of Theorems 1.2-1.4, see lines +3 and -2 on [1, page 1671], line +16 on [1, page 1672]. By these facts, the main results of [1] must be reconsidered.

In this note we give a counterexample to show that Theorem 1.1 and Theorem 1.2 above, which are results for cyclic quasi-contractions, are not true. Then so are not the proofs of Theorem 1.3 and Theorem 1.4. However we do not know whether the conclusions of Theorem 1.3 and Theorem 1.4, which are results for cyclic strongly quasi-contractions, hold or not. So the Question 1.1 is redundant if the conclusions of Theorem 1.3 and Theorem 1.4 do not hold.

\section{MAin Results}

First we give a map $T$ and a uniformly convex Banach space that satisfy all assumptions of Theorem 1.1 and Theorem 1.2 but the conclusions of Theorem 1.1 and Theorem 1.2 do not hold. The map $T$ and the space $X$ also satisfy all assumptions of [12, Theorem 4.1] but $T$ is fixed point free, that is, the conclusion of [12, Theorem 4.1] does not hold.

Example 2.1. Let $X=\mathbb{R}^{2}$ with the Euclidean norm, and

$$
M=(0,0), \quad N=(2,0), \quad P=(2,1), \quad Q=(0,1), \quad I=\left(1, \frac{1}{2}\right),
$$

and $A=[M, P], B=\{N, Q\}$, and $T: A \cup B \longrightarrow A \cup B$ be defined by

$$
T x= \begin{cases}N, & \text { if } x \in[M, I], \\ Q, & \text { if } x \in(I, P],\end{cases}
$$

and $T N=P, T Q=M$. Then

(a) $X$ is a uniformly convex Banach space;

(b) $A$ and $B$ are nonempty subsets of $X, A$ is convex and $T A \subset B, T B \subset A$;

(c) $T$ is a cyclic quasi-contraction;

(d) There exists $x_{0} \in A \cup B$ and $x_{n+1}=T x_{n}$ for all $n \geq 0$ such that

$$
\lim _{n \rightarrow \infty} d\left(x_{n}, x_{n+1}\right) \neq d(A, B), \quad \lim _{n \rightarrow \infty}\left\|x_{2 n+2}-x_{2 n}\right\| \neq 0
$$

and

$$
\lim _{n \rightarrow \infty}\left\|x_{2 n+3}-x_{2 n+1}\right\| \neq 0
$$

(e) $T$ and $T^{2}$ are fixed point free.

Proof. (a), (b) and (e) are trivial.

(c). Let $x \in A$ and $y \in B$. We consider the following four cases.

Case 1. $x \in[M, I], y=N$. We have

$$
d(T x, T y)=d(N, P)=1, \quad d(x, T y)=d(x, P) \geq \frac{\sqrt{5}}{2} .
$$


So $d(T x, T y) \leq \frac{2}{\sqrt{5}} d(x, T y)$.

Case 2. $x \in[M, I], y=Q$. We have

$$
d(T x, T y)=d(N, M)=2, \quad d(y, T x)=d(Q, N)=\sqrt{5} .
$$

So $d(T x, T y)=\frac{2}{\sqrt{5}} d(y, T x)$.

Case 3. $x \in(I, P], y=N$. We have

$$
d(T x, T y)=d(Q, P)=2, \quad d(y, T x)=d(N, Q)=\sqrt{5} .
$$

So $d(T x, T y)=\frac{2}{\sqrt{5}} d(y, T x)$.

Case 4. $x \in(I, P], y=Q$. We have

$$
d(T x, T y)=d(Q, M)=1, \quad d(x, T y)=d(x, M) \geq \frac{\sqrt{5}}{2} .
$$

So $d(T x, T y) \leq \frac{2}{\sqrt{5}} d(x, T y)$.

By the above four cases we find that (1.1) holds for all $x \in A, y \in B$ and for some $c \in\left[\frac{2}{\sqrt{5}}, 1\right)$. So $T$ is a cyclic quasi-contraction.

(d). For $x_{0}=M \in A$ we find that $x_{1}=N, x_{2}=P, x_{3}=Q, x_{4}=M, \ldots, x_{4 n}=M$, $x_{4 n+1}=N, x_{4 n+2}=P, x_{4 n+3}=Q, \ldots$

Then $\lim _{n \rightarrow \infty} d\left(x_{4 n}, x_{4 n+1}\right)=d(M, N)=2$ and

$$
\lim _{n \rightarrow \infty} d\left(x_{4 n+1}, x_{4 n+2}\right)=d(N, P)=1 .
$$

So $\lim _{n \rightarrow \infty} d\left(x_{n}, x_{n+1}\right)$ does not exist. This implies that $\lim _{n \rightarrow \infty} d\left(x_{n}, x_{n+1}\right) \neq d(A, B)$.

We also have

$$
\lim _{n \rightarrow \infty}\left\|x_{2 n+2}-x_{2 n}\right\|=d(P, M)=\sqrt{5} \neq 0,
$$

and

$$
\lim _{n \rightarrow \infty}\left\|x_{2 n+3}-x_{2 n+1}\right\|=d(Q, N)=\sqrt{5} \neq 0 .
$$

Next we revise Theorem 1.1 by replacing the value

$$
\max \{d(x, y), d(x, T x), d(y, T y), d(x, T y), d(y, T x)\}
$$

in (1.1) by

$$
\max \left\{d(x, y), d(x, T x), d(y, T y), \frac{d(x, T y)+d(y, T x)}{2}\right\}
$$

as follows.

Theorem 2.1. Let $A$ and $B$ be nonempty subsets of a metric space $X$ and let $T$ : $A \cup B \longrightarrow A \cup B$ be a map such that for all $x \in A, y \in B$ and some $c \in[0,1)$,

$$
\begin{aligned}
d(T x, T y) \leq & c \max \left\{d(x, y), d(x, T x), d(y, T y), \frac{d(x, T y)+d(y, T x)}{2}\right\} \\
& +(1-c) d(A, B) .
\end{aligned}
$$


For $x_{0} \in A \cup B$, define $x_{n+1}=T x_{n}$ for each $n \geq 0$. Then $\lim _{n \rightarrow \infty} d\left(x_{n}, x_{n+1}\right)=$ $d(A, B)$.

Proof. Without loss of generality we may assume that $x_{0} \in A$. Then $x_{1} \in B, x_{2} \in A$, $\ldots, x_{2 n} \in A, x_{2 n+1} \in B, \ldots$ By the symmetry of $x$ and $y$ in (2.1) we find that (2.1) holds for $x=x_{n}$ and $y=x_{n+1}$ for all $n$. Therefore

$$
\begin{aligned}
& d\left(x_{n+1}, x_{n+2}\right) \\
= & d\left(T x_{n}, T x_{n+1}\right) \\
\leq & c \max \left\{d\left(x_{n}, x_{n+1}\right), d\left(x_{n}, T x_{n}\right), d\left(x_{n+1}, T x_{n+1}\right), \frac{d\left(x_{n}, T x_{n+1}\right)+d\left(x_{n+1}, T x_{n}\right)}{2}\right\} \\
& +(1-c) d(A, B) \\
= & c \max \left\{d\left(x_{n}, x_{n+1}\right), d\left(x_{n}, x_{n+1}\right), d\left(x_{n+1}, x_{n+2}\right), \frac{d\left(x_{n}, x_{n+2}\right)+d\left(x_{n+1}, x_{n+1}\right)}{2}\right\} \\
& +(1-c) d(A, B) \\
= & c \max \left\{d\left(x_{n}, x_{n+1}\right), d\left(x_{n}, x_{n+1}\right), d\left(x_{n+1}, x_{n+2}\right), \frac{d\left(x_{n}, x_{n+2}\right)}{2}\right\} \\
& +(1-c) d(A, B) \\
\leq & c \max \left\{d\left(x_{n}, x_{n+1}\right), d\left(x_{n}, x_{n+1}\right), d\left(x_{n+1}, x_{n+2}\right), \frac{d\left(x_{n}, x_{n+1}\right)+d\left(x_{n+1}, x_{n+2}\right)}{2}\right\} \\
& +(1-c) d(A, B) \\
= & c \max \left\{d\left(x_{n}, x_{n+1}\right), d\left(x_{n+1}, x_{n+2}\right)\right\}+(1-c) d(A, B) .
\end{aligned}
$$

Note that $d\left(x_{n+1}, x_{n+2}\right) \geq d(A, B)$ for all $n$. If $d\left(x_{n+1}, x_{n+2}\right)=d(A, B)$ then $d\left(x_{n+1}, x_{n+2}\right) \leq d\left(x_{n}, x_{n+1}\right)$. If $d\left(x_{n+1}, x_{n+2}\right)>d(A, B)$ then from (2.2) we get

$$
\begin{aligned}
d\left(x_{n+1}, x_{n+2}\right) & <c \max \left\{d\left(x_{n}, x_{n+1}\right), d\left(x_{n+1}, x_{n+2}\right)\right\}+(1-c) d\left(x_{n+1}, x_{n+2}\right) \\
& \leq \max \left\{d\left(x_{n}, x_{n+1}\right), d\left(x_{n+1}, x_{n+2}\right)\right\} .
\end{aligned}
$$

This implies that $d\left(x_{n+1}, x_{n+2}\right)<d\left(x_{n}, x_{n+1}\right)$.

So we have $d\left(x_{n+1}, x_{n+2}\right) \leq d\left(x_{n}, x_{n+1}\right)$ for all $n$, that is, the sequence $\left\{d\left(x_{n}, x_{n+1}\right)\right\}$ is decreasing. Then there exists $\lim _{n \rightarrow \infty} d\left(x_{n}, x_{n+1}\right)=l \geq 0$. Note that $l \geq d(A, B)$. Suppose that $l>d(A, B)$. Letting $n \rightarrow \infty$ in (2.2) we get

$$
l \leq c \max \{l, l\}+(1-c) d(A, B)<c l+(1-c) l=l .
$$

This is a contradiction. Then $l=d(A, B)$ and that $\lim _{n \rightarrow \infty} d\left(x_{n}, x_{n+1}\right)=d(A, B)$. 
Note that for the map $T$ and $x=P, y=N$ and $A, B$ as in Example 2.1 we have

$$
\begin{aligned}
d(T x, T y) & =d(Q, P)=2, & d(x, y) & =d(P, N)=1, \\
d(x, T x) & =d(P, Q)=2, & d(y, T y) & =d(N, P)=1 \\
\frac{d(x, T y)+d(y, T x)}{2} & =\frac{d(P, P)+d(N, Q)}{2}=\frac{\sqrt{5}}{2}, & d(A, B) & =\frac{2}{\sqrt{5}} .
\end{aligned}
$$

This implies that for all $c \in[0,1)$,

$$
\begin{aligned}
d(T x, T y)> & c \max \left\{d(x, y), d(x, T x), d(y, T y), \frac{d(x, T y)+d(y, T x)}{2}\right\} \\
& +(1-c) d(A, B) .
\end{aligned}
$$

So $T$ does not satisfy the condition (2.1).

Now by using Theorem 2.1 playing the role of Theorem 1.1 in the proofs of Theorem 1.2, Theorem 1.3 and Theorem 1.4 in [1] we get the revisions of Theorem 1.2, Theorem 1.3 and Theorem 1.4 as follows.

Theorem 2.2. Let $A$ and $B$ be nonempty subsets of a uniformly convex Banach space $X$ and let $T: A \cup B \longrightarrow A \cup B$ be a map such that $A$ is convex and for all $x \in A, y \in B$ and some $c \in[0,1)$ the condition (2.1) holds. For $x_{0} \in A \cup B$, define $x_{n+1}=T x_{n}$ for each $n \geq 0$. Then $\lim _{n \rightarrow \infty}\left\|x_{2 n+2}-x_{2 n}\right\|=0$ and $\lim _{n \rightarrow \infty}\left\|x_{2 n+3}-x_{2 n+1}\right\|=0$.

Theorem 2.3. Let $A$ and $B$ be nonempty subsets of a uniformly convex Banach space $X$ and let $T: A \cup B \longrightarrow A \cup B$ be a map such that $A$ is convex and for all $x \in A$, $y \in B$ and some $c \in[0,1)$ the conditions (2.1) and (1.2) hold. For $x_{0} \in A \cup B$, define $x_{n+1}=T x_{n}$ for each $n \geq 0$. Then for each $\varepsilon>0$ there exists $n_{0}$ such that for all $m>n \geq n_{0}$ we have $\left\|x_{2 m}-x_{2 n+1}\right\|<d(A, B)+\varepsilon$.

Theorem 2.4. Let $A$ and $B$ be nonempty subsets of a uniformly convex Banach space $X$ and let $T: A \cup B \longrightarrow A \cup B$ be a map such that $A$ and $B$ are convex and for all $x \in A, y \in B$ and some $c \in[0,1)$ the conditions (2.1) and (1.2) hold. For $x_{0} \in A \cup B$, define $x_{n+1}=T x_{n}$ for each $n \geq 0$. Then there exists unique $x \in A$ such that $\lim _{n \rightarrow \infty} x_{2 n}=x, T^{2} x=x$ and $\|x-T x\|=d(A, B)$.

Note that Example 2.1 may not applicable to show that Theorem 1.3 and Theorem 1.4 are incorrect since $T$ does not satisfy (1.2) and $B$ is not convex. So the following question remains open.

Question 2.1. Prove or disprove Theorem 1.3 and Theorem 1.4.

If Theorem 1.4 is proved then we may ask again the question of Amini-Harandi in [1].

Question 2.2 ([1], Question 2.8). Does the conclusion of Theorem 1.4 remains true for cyclic quasi-contraction maps? 
Acknowledgements. The authors gratefully acknowledge the reviewers for their helpful comments.

\section{REFERENCES}

[1] A. Amini-Harandi, Best proximity point theorems for cyclic strongly quasi-contraction mappings, J. Glob. Optim. 56 (2013), 1667-1674.

[2] T. V. An, N. V. Dung, Z. Kadelburg and S. Radenović, Various generalizations of metric spaces and fixed point theorems, Rev. R. Acad. Cienc. Exactas Fís. Nat. Ser. A Mat. RACSAM 109 (2015), 175-198.

[3] S. S. Basha, Best proximity point theorems: resolution of an important non-linear programming problem, Optim. Lett. 7 (2013), 1167-1177.

[4] S. S. Basha, N. Shahzad and R. Jeyaraj, Best proximity points: approximation and optimization, Optim. Lett. 7 (2013), 145-155.

[5] C. M. Chen, Fixed point theorems for cyclic meir-keeler type mappings in complete metric spaces, Fixed Point Theory Appl. 2012:41 (2012), 1-13.

[6] N. V. Dung and V. T. L. Hang, Remarks on cyclic contractions in b-metric spaces and applications to integral equations, Rev. R. Acad. Cienc. Exactas Fís. Nat. Ser. A Mat. RACSAM (2016), 1-9.

[7] E. Karapinar, Fixed point theory for cyclic weak $\phi$-contraction, Appl. Math. Lett. 24 (2011), $822-825$.

[8] E. Karapinar and K. Sadarangani, Corrigendum to "fixed point theory for cyclic weak $\phi$ contraction" [appl. math. lett. 24 (6) (2011) 822-825], Appl. Math. Lett. 25 (2011), 1582-1584.

[9] W. Kirk and N. Shahzad, Fixed Point Theory in Distance Spaces, Springer, Cham, 2014.

[10] W. A. Kirk and B. Sims, Handbook of Metric Fixed Point Theory, Springer Science+Business Media, Dordrecht, 2001.

[11] W. A. Kirk, P. S. Srinivasan and P. Veeramani, Fixed points for mappings satisfying cyclical contractive conditions, Fixed Point Theory 4 (2003), 79-89.

[12] H. K. Nashine, Z. Kadelburg and S. Radenović, Fixed point theorems via various cyclic contractive conditions in partial metric spaces, Publ. Inst. Math. (Beograd) (N.S.) 93 (2013), 69-93.

[13] M. A. Petric, Some results concerning cyclical contractive mappings, Gen. Math. 18 (2010), 213-226.

[14] V. Pragadeeswarar and M. Marudai, Best proximity points: approximation and optimization in partially ordered metric spaces, Optim. Lett. 7 (2013), 1883-1892.

[15] M. Păcurar and I. A. Rus, Fixed point theory for cyclic $\varphi$-contractions, Nonlinear Anal. 72 (2010), 1181-1187.

[16] S. Radenović, A note on fixed point theory for cyclic $\varphi$-contractions, Fixed Point Theory Appl. 2015:189 (2015), 1-9.

\footnotetext{
${ }^{1}$ Institute of Research And Development,

DUY TAN UNIVERSITY,

R.809, K7/25 Quang Trung,

DA NANG CiTY,

VIET NAM
} 
${ }^{2}$ Faculty of Mathematics and Information Technology Teacher Educhtion, Dong Thap University,

CaO Lanh City,

Dong Thap Province,

VIET NAM

E-mail address: nvdung@dthu.edu.vn

${ }^{3}$ Nonlinear Analysis Research Group,

Ton Duc Thang University,

Ho Chi Minh City,

VIET NAM

${ }^{4}$ Faculty of Mathematics and Statistics,

Ton Duc Thang University,

Ho Chi Minh City,

VIET NAM

E-mail address: stojan.radenovic@tdt.edu.vn

${ }^{*}$ Corresponding AUthor 